Supporting materials for

\title{
Syntheses of $\mathrm{La}_{1-\mathrm{x}} \mathrm{Ba}_{\mathrm{x}} \mathrm{Mn}_{2} \mathrm{Al}_{10} \mathrm{O}_{19}$ Catalysts $(\mathrm{x}=\mathbf{0 , 0 . 0 5})$ in a Novel Microemulsion of Water/iso-Propanol/n-Butanol and Their High Activities of Methane
}

\section{Combustion}

Fei Teng, Shuhui Liang, Buergen Gauge, Yongfa Zhu*

Department of Chemistry, Tsinghua University, Beijing 100084, China

$$
\text { Jinguang } \mathrm{Xu}
$$

Institute of Applied Catalysis, Yantai University, Yantai 264005

Guodong Wen, Zhijian Tian, Zheming Wang, Xiaomei Yang, Guoxing Xiong

State Key Laboratory of Catalysis, Dalian Institute of Chemical Physics, Chinese

Academy of Sciences, Dalian 116023, China

The supporting materials contain 4 figures

\footnotetext{
* Corresponding author. Tel /Fax: +86-10-62787601.

Email: zhuyf@mail.tsinghua.edu.cn
} 

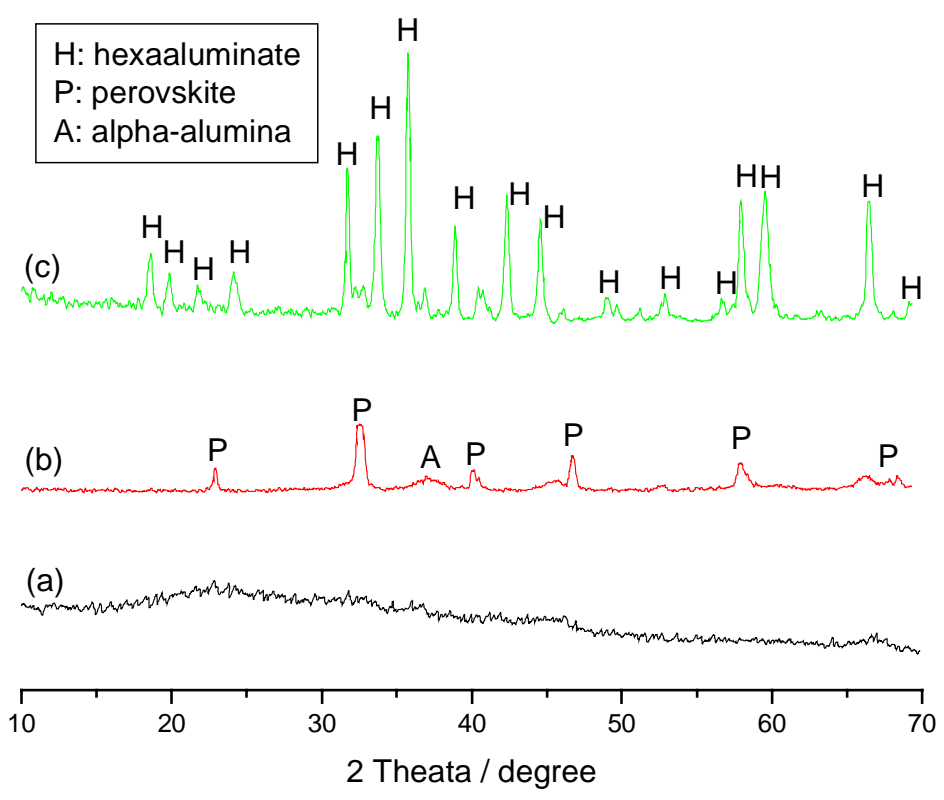

Fig. S1 XRD patterns of $\mathrm{La}_{0.95} \mathrm{Ba}_{0.05} \mathrm{Mn}_{2} \mathrm{Al}_{10} \mathrm{O}_{19}$ catalysts prepared in $\mathrm{W} / \mathrm{iP} / \mathrm{nB}$ dried in oven drying (CD): (a) $110{ }^{\circ} \mathrm{C}, 24 \mathrm{~h}$; (8) $800{ }^{\circ} \mathrm{C}, 2 \mathrm{~h}$; (c) $1200{ }^{\circ} \mathrm{C}, 2 \mathrm{~h} ; \mathrm{M}(\mathrm{OR})_{\mathrm{x}} /$ water $=$ 1/100 (mole ratio), $\mathrm{W} / \mathrm{iP} / \mathrm{nB}=12.5: 12.5: 37.5$ (volume ratios)

The catalyst dried by CD has a lower surface area of $30.4 \mathrm{~m}^{2} \mathrm{~g}^{-1}$ than that $\left(51.7 \mathrm{~m}^{2} \mathrm{~g}^{-1}\right)$ of the catalyst by subcritical drying (SCD). 

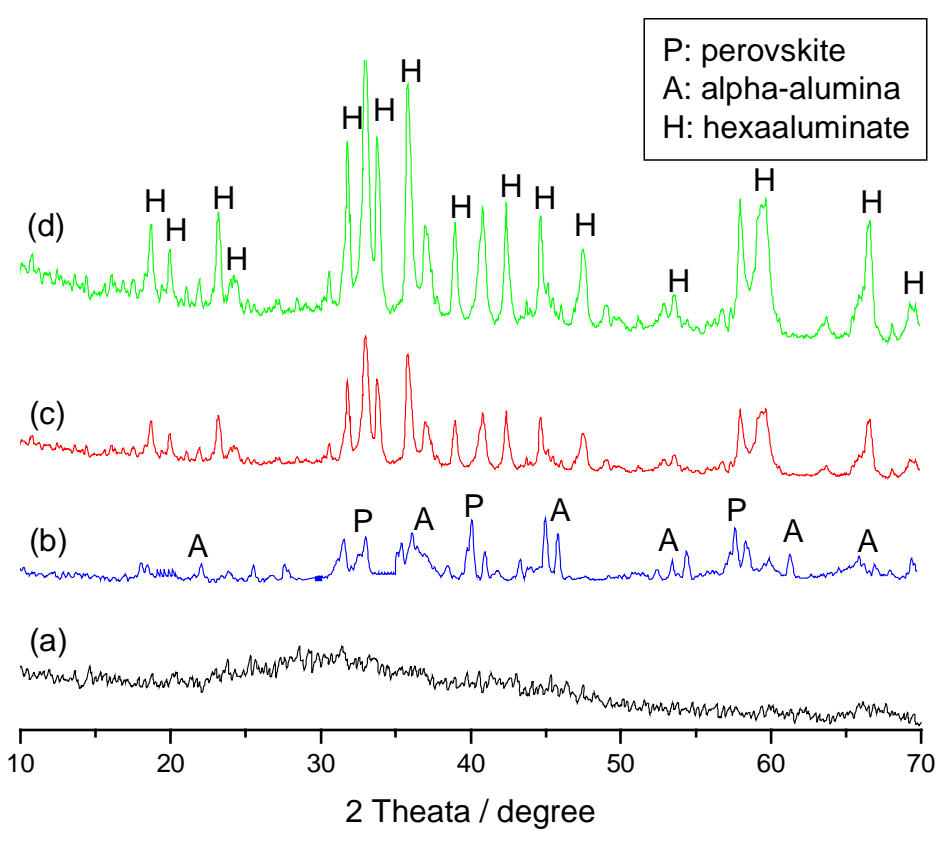

Fig. S2 XRD patterns of $\mathrm{La}_{0.95} \mathrm{Ba}_{0.05} \mathrm{Mn}_{2} \mathrm{Al}_{10} \mathrm{O}_{19}$ catalysts prepared in pure water by conventional oven drying (CD): (a) $110{ }^{\circ} \mathrm{C}, 24 \mathrm{~h}$; (8) $800{ }^{\circ} \mathrm{C}, 2 \mathrm{~h}$; (c) $1200{ }^{\circ} \mathrm{C}, 2 \mathrm{~h}$; (d) $1200{ }^{\circ} \mathrm{C}, 12 \mathrm{~h} ; \mathrm{M}(\mathrm{OR})_{\mathrm{x}} /$ water $=1 / 100$ (mole ratio)

The catalyst dried by CD has a low surface area of $16.1 \mathrm{~m}^{2} \mathrm{~g}^{-1}$. 


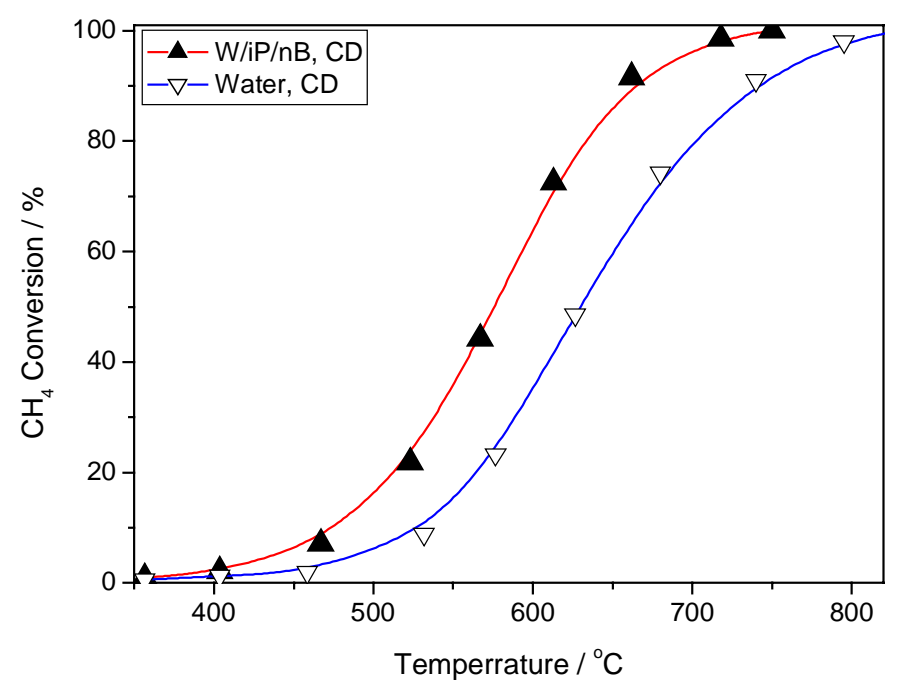

Fig. S3 XRD patterns of $\mathrm{La}_{0.95} \mathrm{Ba}_{0.05} \mathrm{Mn}_{2} \mathrm{Al}_{10} \mathrm{O}_{19}$ catalysts prepared under diferent conditions

$\mathrm{M}(\mathrm{OR})_{\mathrm{x}} /$ water $=1 / 100$ (mole ratio), $\mathrm{W} / \mathrm{iP} / \mathrm{nB}=12.5: 12.5: 37.5$ (volume ratios); calcined at $1200{ }^{\circ} \mathrm{C}, 2 \mathrm{~h}$ 


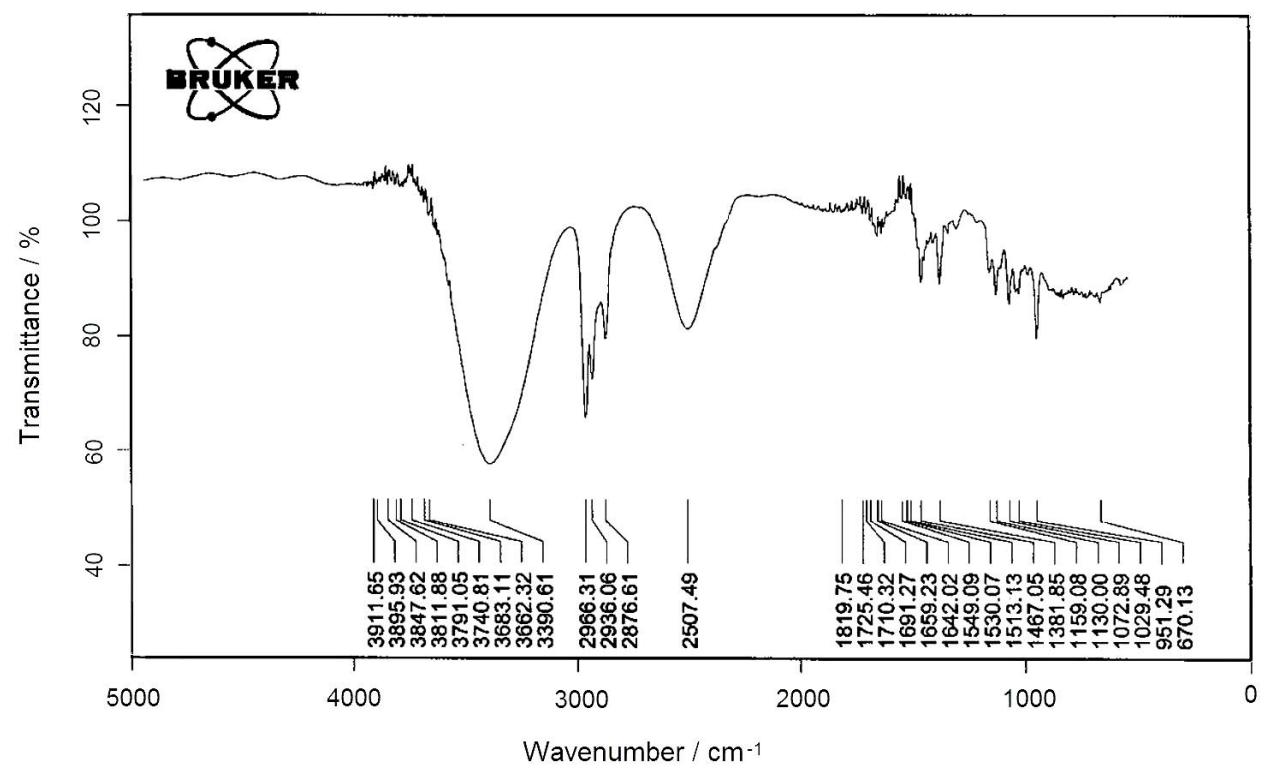

Fig. S4 IR spectra of pure water: Measurement at $25{ }^{\circ} \mathrm{C}, \mathrm{CaF}$ crystal as lens, MCT Detector

$v_{\mathrm{O}-\mathrm{H}}=2507.49 \mathrm{~cm}^{-1}$ 